\title{
Theory of fractional hybrid differential equations with linear perturbations of second type
}

\author{
Hongling Lu, Shurong Sun*, Dianwu Yang and Houshan Teng
}

${ }^{\text {"Correspondence: }}$ sshrong@163.com School of Mathematical Sciences, University of Jinan, Jinan, Shandong 250022, P.R. China

\begin{abstract}
In this paper, we develop the theory of fractional hybrid differential equations with linear perturbations of second type involving Riemann-Liouville differential operators of order $0<q<1$. An existence theorem for fractional hybrid differential equations is proved under the $\varphi$-Lipschitz condition. Some fundamental fractional differential inequalities which are utilized to prove the existence of extremal solutions are also established. Necessary tools are considered and the comparison principle which will be useful for further study of qualitative behavior of solutions is proved.
\end{abstract}

MSC: 34A40; 34A12; 34A99

Keywords: fractional differential inequalities; existence theorem; comparison principle

\section{Introduction}

Fractional differential equations have been of great interest recently. It is caused both by the intensive development of the theory of fractional calculus itself and by the applications; see [1-14]. Although the tools of fractional calculus have been available and applicable to various fields of study, there are few papers on the investigation of the theory of fractional differential equations; see [15-19]. The differential equations involving Riemann-Liouville differential operators of fractional order $0<q<1$ are very important in modeling several physical phenomena [20-22] and therefore seem to deserve an independent study of their theory parallel to the well-known theory of ordinary differential equations.

In recent years, quadratic perturbations of nonlinear differential equations have attracted much attention. The importance of the investigations of hybrid differential equations lies in the fact that they include several dynamic systems as special cases. This class of hybrid differential equations includes the perturbations of original differential equations in different ways. There have been many works on the theory of hybrid differential equations, and we refer the readers to the articles [23-29]. Dhage and Lakshmikantham [24] discussed the following first-order hybrid differential equation with linear perturbations of first type:

$$
\left\{\begin{array}{l}
\frac{\mathrm{d}}{\mathrm{d} t}\left[\frac{x(t)}{f(t, x(t))}\right]=g(t, x(t)), \quad \text { a.e. } t \in J, \\
x\left(t_{0}\right)=x_{0} \in \mathbb{R},
\end{array}\right.
$$

(0) 2013 Lu et al.; licensee Springer. This is an Open Access article distributed under the terms of the Creative Commons Attribution License (http://creativecommons.org/licenses/by/2.0), which permits unrestricted use, distribution, and reproduction in any medium, provided the original work is properly cited. 
where $f \in C(J \times \mathbb{R}, \mathbb{R} \backslash\{0\})$ and $g \in \mathcal{C}(J \times \mathbb{R}, \mathbb{R})$. Dhage and Jadhav [25] discussed the following first-order hybrid differential equation with linear perturbations of second type:

$$
\left\{\begin{array}{l}
\frac{\mathrm{d}}{\mathrm{d} t}[x(t)-f(t, x(t))]=g(t, x(t)), \quad t \in J, \\
x\left(t_{0}\right)=x_{0} \in \mathbb{R},
\end{array}\right.
$$

where $f \in C(J \times \mathbb{R}, \mathbb{R} \backslash\{0\})$ and $g \in \mathcal{C}(J \times \mathbb{R}, \mathbb{R})$. They established the existence and uniqueness results and some fundamental differential inequalities for hybrid differential equations initiating the study of theory of such systems and proved utilizing the theory of inequalities, its existence of extremal solutions and a comparison result.

From the above works, we develop the theory of fractional hybrid differential equations involving Riemann-Liouville differential operators of order $0<q<1$. In this paper, we initiate the basic theory of fractional hybrid differential equations of mixed perturbations of second type involving three nonlinearities and prove the basic result such as the strict and nonstrict fractional differential inequalities, an existence theorem and maximal and minimal solutions etc. We claim that the results of this paper are a basic and important contribution to the theory of nonlinear fractional differential equations.

\section{Fractional hybrid differential equation}

Let $\mathbb{R}$ be a real line and $J=\left[t_{0}, t_{0}+a\right)$ be a bounded interval in $\mathbb{R}$ for some $t_{0}, a \in \mathbb{R}$ with $a>0$. Let $C(J \times \mathbb{R}, \mathbb{R})$ denote the class of continuous functions $f: J \times \mathbb{R} \rightarrow \mathbb{R}$.

Definition 2.1 [19] The Riemann-Liouville fractional derivative of order $\alpha>0$ of a continuous function $f:\left(t_{0},+\infty\right) \rightarrow \mathbb{R}$ is given by

$$
D^{\alpha} f(t)=\frac{1}{\Gamma(n-\alpha)}\left(\frac{d}{d t}\right)^{(n)} \int_{t_{0}}^{t} \frac{f(s)}{(t-s)^{\alpha-n+1}} d s
$$

where $n=[\alpha]+1,[\alpha]$ denotes the integer part of number $\alpha$, provided that the right-hand side is pointwise defined on $\left(t_{0},+\infty\right)$.

Definition 2.2 [19] The Riemann-Liouville fractional integral of order $\alpha>0$ of a function $f:(0,+\infty) \rightarrow \mathbb{R}$ is given by

$$
I^{\alpha} f(t)=\frac{1}{\Gamma(\alpha)} \int_{t_{0}}^{t}(t-s)^{\alpha-1} f(s) d s
$$

provided that the right-hand side is pointwise defined on $\left(t_{0},+\infty\right)$.

We consider fractional hybrid differential equations (in short FHDE) involving RiemannLiouville differential operators of order $0<q<1$,

$$
\left\{\begin{array}{l}
D^{q}[x(t)-f(t, x(t))]=g(t, x(t)), \quad t \in J, \\
x\left(t_{0}\right)=x_{0}
\end{array}\right.
$$

where $f, g \in C(J \times \mathbb{R}, \mathbb{R})$. 
By a solution of FHDE (2.1), we mean a function $x \in C(J, \mathbb{R})$ such that

(i) the function $t \mapsto x-f(t, x)$ is continuous for each $x \in \mathbb{R}$, and

(ii) $x$ satisfies the equations in (2.1).

The theory of strict and nonstrict differential inequalities related to ODEs and hybrid differential equations is available in the literature (see $[24,25,28,29])$. It is known that differential inequalities are useful for proving the existence of extremal solutions of ODEs and hybrid differential equations defined on $J$.

\section{Existence result}

In this section, we prove the existence results for $\operatorname{FHDE}(2.1)$ on the closed and bounded interval $J=\left[t_{0}, t_{0}+a\right]$ under mixed Lipschitz and compactness conditions on the nonlinearities involved in it.

We place FHDE (2.1) in the space $C(J, \mathbb{R})$ of continuous real-valued functions defined on $J$. Define a supremum norm $\|\cdot\|$ in $C(J, \mathbb{R})$ by $\|x\|=\sup _{t \in J}|x(t)|$. Clearly, $C(J, \mathbb{R})$ is a Banach algebra with respect to the above norm.

We prove the existence of a solution for FHDE (2.1) by a fixed point theorem in the Banach algebra due to Dhage [30].

Definition 3.1 Let $X$ be a Banach space. A mapping $T: X \rightarrow X$ is called $\varphi$-Lipschitzian if there exists a continuous and nondecreasing function $\varphi: R^{+} \rightarrow R^{+}$such that

$$
\|T x-T y\| \leq \varphi(\|x-y\|)
$$

for all $x, y \in X$, where $\varphi(0)=0$.

Further, if $\varphi$ satisfies the condition $\varphi(r)<r, r>0$, then $T$ is called a nonlinear contraction with a control function $\varphi$.

Lemma 3.1 [30] Let $S$ be a nonempty, closed convex and bounded subset of the Banach algebra $X$ and let $A: X \rightarrow X$ and $B: S \rightarrow X$ be two operators such that
(a) $A$ is nonlinear contraction,
(b) B is completely continuous,
(c) $A x+B x \in S$ for all $x \in S$.

Then the operator equation $A x+B x=x$ has a solution in $S$.

We consider the following hypotheses in what follows.

$\left(\mathrm{A}_{0}\right)$ The function $x \mapsto x-f(t, x)$ is increasing in $\mathbb{R}$ for all $t \in J$.

( $\left.\mathrm{A}_{1}\right)$ There exist constants $M \geq L>0$ such that

$$
|f(t, x)-f(t, y)| \leq \frac{L|x-y|}{M+|x-y|}
$$

for all $t \in J$ and $x, y \in \mathbb{R}$.

$\left(\mathrm{A}_{3}\right)$ There exists a continuous function $h \in C(J, \mathbb{R})$ such that

$$
|g(t, x)| \leq h(t), \quad t \in J
$$

for all $x \in \mathbb{R}$. 
Lemma 3.2 [19] Let $0<q<1$ and $u \in L^{1}(0, T)$.

$\left(\mathrm{H}_{1}\right)$ The equality $D^{q} I^{q} u(t)=u(t)$ holds.

$\left(\mathrm{H}_{2}\right)$ The equality

$$
I^{q} D^{q} u(t)=u(t)-\frac{I^{1-q} u\left(t_{0}\right)}{\Gamma(q)}\left(t-t_{0}\right)^{q-1}
$$

holds almost everywhere on $J$.

The following lemma is useful in what follows.

Lemma 3.3 Assume that hypothesis $\left(\mathrm{A}_{0}\right)$ holds. Then, for any $h \in C(J, \mathbb{R})$ and $0<q<1$, the function $x \in C(J, \mathbb{R})$ is a solution of the FHDE

$$
D^{q}[x(t)-f(t, x(t))]=h(t), \quad t \in J,
$$

and

$$
x\left(t_{0}\right)=x_{0},
$$

if and only if $x$ satisfies the hybrid integral equation (HIE)

$$
x(t)=x_{0}-f\left(t_{0}, x_{0}\right)+f(t, x(t))+\frac{1}{\Gamma(q)} \int_{t_{0}}^{t}(t-s)^{q-1} h(s) d s, \quad t \in J .
$$

Proof Let $x$ be a solution of the Cauchy problem (3.1) and (3.2). Since the RiemannLiouville fractional integral $I^{q}$ is a monotone operator, thus we apply the fractional integral $I^{q}$ on both sides of (3.1). By Lemma 3.2, we have

$$
I^{q} D^{q}[x(t)-f(t, x(t))]=x(t)-f(t, x(t))-\frac{\left.I^{1-q}[x(t)-f(t, x(t))]\right|_{t=t_{0}}}{\Gamma(q)}\left(t-t_{0}\right)^{q-1}=I^{q} h(t),
$$

then by (3.2), we get

$$
x(t)-f(t, x(t))=I^{q} h(t)+\frac{\left.I^{1-q}[x(t)-f(t, x(t))]\right|_{t=t_{0}}}{\Gamma(q)}\left(t-t_{0}\right)^{q-1}=x_{0}-f\left(t_{0}, x_{0}\right)+I^{q} h(t),
$$

i.e.,

$$
\begin{aligned}
x(t) & =x_{0}-f\left(t_{0}, x_{0}\right)+f(t, x(t))+I^{q} h(t) \\
& =x_{0}-f\left(t_{0}, x_{0}\right)+f(t, x(t))+\frac{1}{\Gamma(q)} \int_{t_{0}}^{t}(t-s)^{q-1} h(s) d s, \quad t \in J .
\end{aligned}
$$

Thus, (3.3) holds.

Conversely, assume that $x$ satisfies HIE (3.3). Then applying $D^{q}$ on both sides of (3.3), (3.1) is satisfied. Again, substituting $t=t_{0}$ in (3.3) yields

$$
x\left(t_{0}\right)-f\left(t_{0}, x\left(t_{0}\right)\right)=x_{0}-f\left(t_{0}, x_{0}\right) .
$$


The map $x \mapsto x-f(t, x)$ is increasing in $\mathbb{R}$ for all $t \in J$, the map $x \mapsto x-f\left(t_{0}, x\right)$ is injective in $\mathbb{R}$, hence $x\left(t_{0}\right)=x_{0}$. The proof is completed.

Now, we are in a position to prove the following existence theorem for FHDE (2.1).

Theorem 3.1 Assume that hypotheses $\left(\mathrm{A}_{0}\right)-\left(\mathrm{A}_{2}\right)$ hold. Then FHDE (2.1) has a solution defined on $J$.

Proof Set $X=C(J, \mathbb{R})$ and define a subset $S$ of $X$ defined by

$$
S=\{x \in X \mid\|x\| \leq N\}
$$

where $N=\left|x_{0}-f\left(t_{0}, x_{0}\right)\right|+L+F_{0}+\frac{a^{q}}{\Gamma(q+1)}\|h\|_{L^{1}}$ and $F_{0}=\sup _{t \in J}|f(t, 0)|$.

Clearly, $S$ is a closed, convex and bounded subset of the Banach algebra $X$. Now, using the hypotheses $\left(\mathrm{A}_{0}\right)-\left(\mathrm{A}_{2}\right)$, it can be shown by an application of Lemma 3.3, FHDE (2.1) is equivalent to the nonlinear $\mathrm{HIE}$

$$
x(t)=x_{0}-f\left(t_{0}, x_{0}\right)+f(t, x(t))+\frac{1}{\Gamma(q)} \int_{t_{0}}^{t}(t-s)^{q-1} g(s, x(s)) d s, \quad t \in J .
$$

Define two operators $A: X \rightarrow X$ and $B: S \rightarrow X$ by

$$
A x(t)=f(t, x(t)), \quad t \in J,
$$

and

$$
B x(t)=x_{0}-f\left(t_{0}, x_{0}\right)+\frac{1}{\Gamma(q)} \int_{t_{0}}^{t}(t-s)^{q-1} g(s, x(s)) d s, \quad t \in J .
$$

Then HIE (3.5) is transformed into the operator equation as

$$
A x(t)+B x(t)=x(t), \quad t \in J .
$$

We will show that the operators $A$ and $B$ satisfy all the conditions of Lemma 3.1.

First, we show that $A$ is a Lipschitz operator on $X$ with the Lipschitz constant $L$. Let $x, y \in X$. Then by hypothesis $\left(\mathrm{A}_{1}\right)$,

$$
|A x(t)-A y(t)|=|f(t, x(t))-f(t, y(t))| \leq \frac{L|x(t)-y(t)|}{M+|x(t)-y(t)|} \leq \frac{L\|x-y\|}{M+\|x-y\|}
$$

for all $t \in J$. Taking supremum over $t$, we obtain

$$
\|A x-A y\| \leq \frac{L\|x-y\|}{M+\|x-y\|}
$$

for all $x, y \in X$. This shows that $A$ is a nonlinear contraction on $X$ with a control function $\varphi$ defined by $\varphi=\frac{L r}{M+r}$. 
Next, we show that $B$ is a compact and continuous operator on $S$ into $X$. First, we show that $B$ is continuous on $S$. Let $\left\{x_{n}\right\}$ be a sequence in $S$ converging to a point $x \in S$. Then, by the Lebesgue dominated convergence theorem,

$$
\begin{aligned}
\lim _{n \rightarrow \infty} B x_{n}(t) & =x_{0}-f\left(t_{0}, x_{0}\right)+\lim _{n \rightarrow \infty} \frac{1}{\Gamma(q)} \int_{t_{0}}^{t}(t-s)^{q-1} g\left(s, x_{n}(s)\right) d s \\
& =x_{0}-f\left(t_{0}, x_{0}\right)+\frac{1}{\Gamma(q)} \int_{t_{0}}^{t}(t-s)^{q-1} \lim _{n \rightarrow \infty} g\left(s, x_{n}(s)\right) d s \\
& =x_{0}-f\left(t_{0}, x_{0}\right)+\frac{1}{\Gamma(q)} \int_{t_{0}}^{t}(t-s)^{q-1} g(s, x(s)) d s \\
& =B x(t)
\end{aligned}
$$

for all $t \in J$. This shows that $B$ is a continuous operator on $S$.

Now, we show that $B$ is a compact operator on $S$. It is enough to show that $B(S)$ is a uniformly bounded and equicontinuous set in $X$. On the one hand, let $x \in S$ be arbitrary. Then by hypothesis $\left(\mathrm{A}_{2}\right)$,

$$
\begin{aligned}
|B x(t)| & =\left|x_{0}-f\left(t_{0}, x_{0}\right)\right|+\left|\frac{1}{\Gamma(q)} \int_{t_{0}}^{t}(t-s)^{q-1} g(s, x(s)) d s\right| \\
& \leq\left|x_{0}-f\left(t_{0}, x_{0}\right)\right|+\frac{1}{\Gamma(q)} \int_{t_{0}}^{t}(t-s)^{q-1}|g(s, x(s))| d s \\
& \leq\left|x_{0}-f\left(t_{0}, x_{0}\right)\right|+\frac{1}{\Gamma(q)} \int_{t_{0}}^{t}(t-s)^{q-1} h(s) d s \\
& \leq\left|x_{0}-f\left(t_{0}, x_{0}\right)\right|+\frac{a^{q}}{\Gamma(q+1)}\|h\|_{L^{1}},
\end{aligned}
$$

for all $t \in J$. Taking supremum over $t$,

$$
\|B x\| \leq\left|x_{0}-f\left(t_{0}, x_{0}\right)\right|+\frac{a^{q}}{\Gamma(q+1)}\|h\|_{L^{1}}
$$

for all $x \in S$. This shows that $B$ is uniformly bounded on $S$.

On the other hand, let $t_{1}, t_{2} \in J$ with $t_{1}<t_{2}$. Then, for any $x \in S$, one has

$$
\begin{aligned}
\left|B x\left(t_{1}\right)-B x\left(t_{2}\right)\right|= & \left|\frac{1}{\Gamma(q)} \int_{t_{0}}^{t_{1}}\left(t_{1}-s\right)^{q-1} g(s, x(s)) d s-\frac{1}{\Gamma(q)} \int_{t_{0}}^{t_{2}}\left(t_{2}-s\right)^{q-1} g(s, x(s)) d s\right| \\
\leq & \left|\frac{1}{\Gamma(q)} \int_{t_{0}}^{t_{1}}\left(t_{1}-s\right)^{q-1} g(s, x(s)) d s-\frac{1}{\Gamma(q)} \int_{t_{0}}^{t_{1}}\left(t_{2}-s\right)^{q-1} g(s, x(s)) d s\right| \\
& +\left|\frac{1}{\Gamma(q)} \int_{t_{0}}^{t_{1}}\left(t_{2}-s\right)^{q-1} g(s, x(s)) d s-\frac{1}{\Gamma(q)} \int_{t_{0}}^{t_{2}}\left(t_{2}-s\right)^{q-1} g(s, x(s)) d s\right| \\
\leq & \frac{\|h\|_{L^{1}}}{\Gamma(q+1)}\left[\left|\left(t_{2}-t_{0}\right)^{q}-\left(t_{1}-t_{0}\right)^{q}-\left(t_{2}-t_{1}\right)^{q}\right|+\left(t_{2}-t_{1}\right)^{q}\right] .
\end{aligned}
$$

Hence, for $\varepsilon>0$, there exists a $\delta>0$ such that

$$
\left|t_{1}-t_{2}\right|<\delta \Rightarrow\left|B x\left(t_{1}\right)-B x\left(t_{2}\right)\right|<\varepsilon,
$$


for all $t_{1}, t_{2} \in J$ and for all $x \in S$. This shows that $B(S)$ is an equicontinuous set in $X$. Now, the set $B(S)$ is a uniformly bounded and equicontinuous set in $X$, so it is compact by the Arzela-Ascoli theorem. As a result, $B$ is a complete continuous operator on $S$.

Next, we show that hypothesis (c) of Lemma 3.1 is satisfied. Let $x \in S$. Then, by assumption $\left(\mathrm{A}_{1}\right)$, we have

$$
\begin{aligned}
|A x(t)+B x(t)| \leq & |A x(t)|+|B x(t)| \\
\leq & \left|x_{0}-f\left(t_{0}, x_{0}\right)\right|+|f(t, x(t))|+\left|\frac{1}{\Gamma(q)} \int_{t_{0}}^{t}(t-s)^{q-1} g(s, y(s)) d s\right| \\
\leq & \left|x_{0}-f\left(t_{0}, x_{0}\right)\right|+[|f(t, x(t))-f(t, 0)|+|f(t, 0)|] \\
& \quad+\left(\frac{1}{\Gamma(q)} \int_{t_{0}}^{t}(t-s)^{q-1}|g(s, x(s))| d s\right) \\
\leq & \left|x_{0}-f\left(t_{0}, x_{0}\right)\right|+L+F_{0}+\left(\frac{1}{\Gamma(q)} \int_{t_{0}}^{t}(t-s)^{q-1} h(s) d s\right) \\
\leq & \left|x_{0}-f\left(t_{0}, x_{0}\right)\right|+L+F_{0}+\frac{T^{q}}{\Gamma(q+1)}\|h\|_{L^{1}} .
\end{aligned}
$$

Taking supremum over $t$,

$$
\|x\| \leq\left|x_{0}-f\left(t_{0}, x_{0}\right)\right|+L+F_{0}+\frac{T^{q}}{\Gamma(q+1)}\|h\|_{L^{1}}=N .
$$

Thus, all the conditions of Lemma 3.1 are satisfied and hence the operator equation $A x+B x=x$ has a solution in $S$. As a result, FHDE (2.1) has a solution defined on $J$. This completes the proof.

\section{Fractional hybrid differential inequalities}

We discuss a fundamental result relative to strict inequalities for FHDE (2.1).

Lemma 4.1 [17] Let $m: \mathbb{R}_{+} \rightarrow \mathbb{R}$ be locally Hölder continuous such that for any $t_{1} \in$ $(0,+\infty)$, we have

$$
m\left(t_{1}\right)=0 \text { and } m(t) \leq 0 \quad \text { for } t_{0} \leq t \leq t_{1} .
$$

Then it follows that

$$
D^{q} m\left(t_{1}\right) \geq 0 .
$$

Theorem 4.1 Assume that hypothesis $\left(\mathrm{A}_{0}\right)$ holds. Suppose that there exist functions $y, z$ : $J \rightarrow \mathbb{R}$ that are locally Hölder continuous such that

$$
D^{q}[y(t)-f(t, y(t))] \leq g(t, y(t)), \quad t \in J,
$$

and

$$
D^{q}[z(t)-f(t, z(t))] \geq g(t, z(t)), \quad t \in J,
$$


one of the inequalities being strict. Then

$$
y\left(t_{0}\right)<z\left(t_{0}\right)
$$

implies

$$
y(t)<z(t)
$$

for all $t \in J$.

Proof Suppose that inequality (4.4) is strict. Assume that the claim is false. Then there exists a $t_{1} \in J, t_{1}>0$, such that $y\left(t_{1}\right)=z\left(t_{1}\right)$ and $y(t)<z(t)$ for $t_{0} \leq t<t_{1}$.

Define

$$
Y(t)=y(t)-f(t, y(t)) \quad \text { and } \quad Z(t)=z(t)-f(t, z(t)) .
$$

Then we have $Y\left(t_{1}\right)=Z\left(t_{1}\right)$ and by virtue of hypothesis $\left(\mathrm{A}_{0}\right)$, we get $Y(t)<Z(t)$ for all $t_{0} \leq t<t_{1}$. Setting $m(t)=Y(t)-Z(t), t_{0} \leq t \leq t_{1}$, we find that $m(t) \leq 0, t_{0} \leq t \leq t_{1}$ and $m\left(t_{1}\right)=0$. Then by Lemma 4.1, we have $D^{q} m\left(t_{1}\right) \geq 0$. By (4.3) and (4.4), we obtain

$$
g\left(t_{1}, y\left(t_{1}\right)\right) \geq D^{q} Y\left(t_{1}\right) \geq D^{q} Z\left(t_{1}\right)>g\left(t_{1}, z\left(t_{1}\right)\right) .
$$

This is a contradiction to $y\left(t_{1}\right)=z\left(t_{1}\right)$. Hence, the conclusion (4.6) is valid and the proof is complete.

The next result is concerned with nonstrict fractional differential inequalities which require a kind of one-sided $\varphi$-Lipshitz condition.

Theorem 4.2 Assume that the conditions of Theorem 4.1 hold. Suppose that there exists a real number $M>0$ such that

$$
g\left(t, x_{1}\right)-g\left(t, x_{2}\right) \leq \frac{M}{1+t^{q}}\left(x_{1}-f\left(t, x_{1}\right)\right)-\left(x_{2}-f\left(t, x_{2}\right)\right), \quad t \in J
$$

for all $x_{1}, x_{2} \in \mathbb{R}$ with $x_{1} \geq x_{2}$. Then $y(0) \leq z(0)$ implies, provided $M a^{q} \leq \frac{1}{\Gamma(1-q)}$,

$$
y(t) \leq z(t)
$$

for all $t \in J$.

Proof We set

$$
z_{\varepsilon}(t)-f\left(t, z_{\varepsilon}(t)\right)=z(t)-f(t, z(t))+\varepsilon\left(1+t^{q}\right)
$$

for small $\varepsilon>0$, so that we have

$$
z_{\varepsilon}(t)-f\left(t, z_{\varepsilon}(t)\right)>z(t)-f(t, z(t)) \quad \Rightarrow \quad z_{\varepsilon}(t)>z(t) .
$$


Define $Z_{\varepsilon}(t)=z_{\varepsilon}(t)-f\left(t, z_{\varepsilon}(t)\right)$ and $Z(t)=z(t)-f(t, z(t))$ for $t \in J$. Since

$$
g\left(t, z_{\varepsilon}(t)\right)-g(t, z(t)) \leq \frac{M}{1+t^{q}}\left(\left(z_{\varepsilon}(t)-f\left(t, z_{\varepsilon}(t)\right)\right)-(z(t)-f(t, z(t)))\right)
$$

for all $t \in J$ and $M a^{q} \leq \frac{1}{\Gamma(1-q)}$, one has

$$
\begin{aligned}
D^{q} Z_{\varepsilon}(t) & =D^{q} Z(t)+\varepsilon D^{q}\left(1+t^{q}\right) \\
& \geq g(t, z(t))+\varepsilon\left(\frac{1}{t^{q} \Gamma(1-q)}+\Gamma(1+q)\right) \\
& \geq g\left(t, z_{\varepsilon}(t)\right)-M \varepsilon+\varepsilon \frac{1}{t^{q} \Gamma(1-q)}+\varepsilon \Gamma(1+q) \\
& >g\left(t, z_{\varepsilon}(t)\right) .
\end{aligned}
$$

Also, we have $z_{\varepsilon}(0)>z(0) \geq y(0)$. Hence, by an application of Theorem 4.1 with $z=z_{\varepsilon}$ yields that $y(t)<z_{\varepsilon}(t)$ for all $t \in J$. By the arbitrariness of $\varepsilon>0$, taking the limits as $\varepsilon \rightarrow 0$, we have $y(t) \leq z(t)$ for all $t \in J$. This completes the proof.

Remark 4.1 Let $f(t, x) \equiv 0$ and $g(t, x)=x$. We can easily verify that $f$ and $g$ satisfy the condition (4.7).

\section{Existence of maximal and minimal solutions}

In this section, we prove the existence of maximal and minimal solutions for FHDE (2.1) on $J=\left[t_{0}, t_{0}+a\right]$. We need the following definition in what follows.

Definition 5.1 A solution $r$ of FHDE (2.1) is said to be maximal if for any other solution $x$ to FHDE (2.1), one has $x(t) \leq r(t)$ for all $t \in J$. Similarly, a solution $\rho$ of FHDE (2.1) is said to be minimal if $\rho(t) \leq x(t)$ for all $t \in J$, where $x$ is any solution of FHDE (2.1) on $J$.

We discuss the case of a maximal solution only, as the case of a minimal solution is similar and can be obtained with the same arguments with appropriate modifications. Given an arbitrary small real number $\varepsilon>0$, consider the following initial value problem of FHDE of order $0<q<1$,

$$
\left\{\begin{array}{l}
D^{q}[x(t)-f(t, x(t))]=g(t, x(t))+\varepsilon, \quad \text { a.e. } t \in J \\
x\left(t_{0}\right)=x_{0}+\varepsilon
\end{array}\right.
$$

where $f, g \in C(J \times \mathbb{R}, \mathbb{R})$.

An existence theorem for FHDE (5.1) can be stated as follows.

Theorem 5.1 Assume that hypotheses $\left(\mathrm{A}_{0}\right)-\left(\mathrm{A}_{2}\right)$ hold. Then, for every small number $\varepsilon>0$, FHDE (5.1) has a solution defined on J.

Proof The proof is similar to Theorem 3.1 and we omit the details.

Our main existence theorem for a maximal solution for FHDE (2.1) is as follows. 
Theorem 5.2 Assume that hypotheses $\left(\mathrm{A}_{0}\right)-\left(\mathrm{A}_{2}\right)$ hold. Then FHDE (2.1) has a maximal solution defined on $J$.

Proof Let $\left\{\varepsilon_{n}\right\}_{0}^{\infty}$ be a decreasing sequence of positive real numbers such that $\lim _{n \rightarrow \infty} \varepsilon_{n}=$ 0 . By Theorem 5.1, then there exists a solution $r\left(t, \varepsilon_{n}\right)$ of the FHDE defined on $J$

$$
\left\{\begin{array}{l}
D^{q}[x(t)-f(t, x(t))]=g(t, x(t))+\varepsilon_{n}, \quad t \in J, \\
x\left(t_{0}\right)=x_{0}+\varepsilon_{n} .
\end{array}\right.
$$

Then, for any solution $u$ of FHDE (2.1), any solution of auxiliary problem (5.2) satisfies

$$
D^{q}\left[r\left(t, \varepsilon_{n}\right)-f\left(t, r\left(t, \varepsilon_{n}\right)\right)\right]=g\left(t, r\left(t, \varepsilon_{n}\right)\right)+\varepsilon_{n}>g\left(t, r\left(t, \varepsilon_{n}\right)\right),
$$

where $u\left(t_{0}\right)=x_{0} \leq x_{0}+\varepsilon_{n}=r\left(t_{0}, \varepsilon_{n}\right)$. By Theorem 4.2, we infer that

$$
u(t) \leq r\left(t, \varepsilon_{n}\right)
$$

for all $t \in J$ and $n \in \mathbb{N} \cup\{0\}$.

Since $x_{0}+\varepsilon_{2}=r\left(t_{0}, \varepsilon_{2}\right) \leq r\left(t_{0}, \varepsilon_{1}\right)=x_{0}+\varepsilon_{1}$, then by Theorem 4.2, we infer that $r\left(t, \varepsilon_{2}\right) \leq$ $r\left(t, \varepsilon_{1}\right)$. Therefore, $r\left(t, \varepsilon_{n}\right)$ is a decreasing sequence of positive real numbers, the limit

$$
r(t)=\lim _{n \rightarrow \infty} r\left(t, \varepsilon_{n}\right)
$$

exists. We show that the convergence in (5.4) is uniform on $J$. To finish, it is enough to prove that the sequence $r\left(t, \varepsilon_{n}\right)$ is equicontinuous in $C(J, R)$. Let $t_{1}, t_{2} \in J$ with $t_{1}<t_{2}$ be arbitrary. Then

$$
\begin{aligned}
\left|r\left(t_{1}, \varepsilon_{n}\right)-r\left(t_{2}, \varepsilon_{n}\right)\right|= & \mid\left(f\left(t_{1}, r\left(t_{1}, \varepsilon_{n}\right)\right)+\frac{1}{\Gamma(q)} \int_{t_{0}}^{t_{1}}\left(t_{1}-s\right)^{q-1}\left(g\left(s, r\left(s, \varepsilon_{n}\right)\right)+\varepsilon_{n}\right) d s\right) \\
& -\left(f\left(t_{2}, r\left(t_{2}, \varepsilon_{n}\right)\right)+\frac{1}{\Gamma(q)} \int_{t_{0}}^{t_{2}}\left(t_{2}-s\right)^{q-1}\left(g\left(s, r\left(s, \varepsilon_{n}\right)\right)+\varepsilon_{n}\right) d s\right) \mid \\
\leq & \left|f\left(t_{1}, r\left(t_{1}, \varepsilon_{n}\right)\right)-f\left(t_{2}, r\left(t_{2}, \varepsilon_{n}\right)\right)\right|+\frac{1}{\Gamma(q)} \int_{t_{1}}^{t_{2}} \varepsilon_{n} d s \\
& +\frac{1}{\Gamma(q)}\left|\int_{t_{0}}^{t_{1}}\left(\left(t_{1}-s\right)^{q-1}-\left(t_{2}-s\right)^{q-1}\right) g\left(s, r\left(s, \varepsilon_{n}\right)\right) d s\right| \\
& +\frac{1}{\Gamma(q)}\left|\int_{t_{1}}^{t_{2}}\left(t_{2}-s\right)^{q-1} g\left(s, r\left(s, \varepsilon_{n}\right)\right) d s\right| \\
\leq & \left|f\left(t_{1}, r\left(t_{1}, \varepsilon_{n}\right)\right)-f\left(t_{2}, r\left(t_{2}, \varepsilon_{n}\right)\right)\right|+\frac{\varepsilon_{n}}{\Gamma(q)}\left(t_{2}-t_{1}\right) \\
& +\frac{\|h\|_{L^{1}}}{\Gamma(q+1)}\left[\left(t_{2}-t_{0}\right)^{q}-\left(t_{1}-t_{0}\right)^{q}\right]+\left(t_{2}-t_{1}\right)^{q} .
\end{aligned}
$$

Since $f$ is continuous on a compact set $J \times[-N, N]$, it is uniformly continuous there. Hence,

$$
\left|f\left(t_{1}, r\left(t_{1}, \varepsilon_{n}\right)\right)-f\left(t_{2}, r\left(t_{2}, \varepsilon_{n}\right)\right)\right| \rightarrow 0 \quad \text { as } t_{1} \rightarrow t_{2}
$$

uniformly for all $n \in \mathbb{N}$. 
Therefore, from the above inequality, it follows that

$$
\left|r\left(t_{1}, \varepsilon_{n}\right)-r\left(t_{2}, \varepsilon_{n}\right)\right| \rightarrow 0 \quad \text { as } t_{1} \rightarrow t_{2}
$$

uniformly for all $n \in \mathbb{N}$. Therefore,

$$
r\left(t, \varepsilon_{n}\right) \rightarrow r(t) \quad \text { as } n \rightarrow \infty
$$

for all $t \in J$.

Next, we show that the function $r(t)$ is a solution of FHDE (2.1) defined on $J$. Now, since $r\left(t, \varepsilon_{n}\right)$ is a solution of FHDE (5.2), we have

$$
r\left(t, \varepsilon_{n}\right)=x_{0}+\varepsilon_{n}+f\left(t, r\left(t, \varepsilon_{n}\right)\right)+\frac{1}{\Gamma(q)} \int_{t_{0}}^{t}(t-s)^{q-1}\left(g\left(s, r\left(s, \varepsilon_{n}\right)\right)+\varepsilon_{n}\right) d s
$$

for all $t \in J$. Taking the limit as $n \rightarrow \infty$ in above Eq. (5.5) yields

$$
r(t)=f(t, r(t))+\frac{1}{\Gamma(q)} \int_{t_{0}}^{t}(t-s)^{q-1} g(s, r(s)) d s
$$

for all $t \in J$. Thus, the function $r$ is a solution of FHDE (2.1) on $J$. Finally, from inequality (5.3), it follows that $u(t) \leq r(t)$ for all $t \in J$. Hence, FHDE (2.1) has a maximal solution on $J$. This completes the proof.

\section{Comparison theorems}

The main problem of differential inequalities is to estimate a bound for the solution set for the differential inequality related to FHDE (2.1). In this section, we prove that the maximal and minimal solutions serve as bounds for the solutions of the related differential inequality to $\operatorname{FHDE}(2.1)$ on $J=\left[t_{0}, t_{0}+a\right]$.

Theorem 6.1 Assume that hypotheses $\left(\mathrm{A}_{0}\right)-\left(\mathrm{A}_{2}\right)$ hold. Suppose that there exists a real number $M>0$ such that

$$
g\left(t, x_{1}\right)-g\left(t, x_{2}\right) \leq \frac{M}{1+t^{q}}\left[\left(x_{1}-f\left(t, x_{1}\right)\right)-\left(x_{2}-f\left(t, x_{2}\right)\right)\right], \quad t \in J,
$$

for all $x_{1}, x_{2} \in \mathbb{R}$ with $x_{1} \geq x_{2}$, where $M a^{q} \leq \frac{1}{\Gamma(1-q)}$. Furthermore, if there exists a function $u \in C(J, \mathbb{R})$ such that

$$
\left\{\begin{array}{l}
D^{q}[u(t)-f(t, u(t))] \leq g(t, u(t)), \quad \text { a.e. } t \in J, \\
u\left(t_{0}\right) \leq x_{0}
\end{array}\right.
$$

Then

$$
u(t) \leq r(t)
$$

for all $t \in J$, where $r$ is a maximal solution of FHDE (2.1) on $J$. 
Proof Let $\varepsilon>0$ be arbitrary small. By Theorem 5.2, $r(t, \varepsilon)$ is a maximal solution of FHDE (5.1) and the limit

$$
r(t)=\lim _{\varepsilon \rightarrow 0} r(t, \varepsilon)
$$

is uniform on $J$ and the function $r$ is a maximal solution of FHDE (2.1) on $J$. Hence, we obtain

$$
\left\{\begin{array}{l}
D^{q}[r(t, \varepsilon)-f(t, r(t, \varepsilon))]=g(t, r(t, \varepsilon))+\varepsilon, \quad t \in J, \\
r\left(t_{0}, \varepsilon\right)=x_{0}+\varepsilon
\end{array}\right.
$$

From the above inequality, it follows that

$$
\left\{\begin{array}{l}
D^{q}[r(t, \varepsilon)-f(t, r(t, \varepsilon))]>g(t, r(t, \varepsilon)), \quad \text { a.e. } t \in J, \\
r\left(t_{0}, \varepsilon\right)>x_{0} .
\end{array}\right.
$$

Now, we apply Theorem 4.2 to inequalities (6.1) and (6.4) and conclude that $u(t)<r(t, \varepsilon)$ for all $t \in J$. This further, in view of limit (6.3), implies that inequality (6.2) holds on $J$. This completes the proof.

Theorem 6.2 Assume that hypotheses $\left(\mathrm{A}_{0}\right)-\left(\mathrm{A}_{2}\right)$ hold. Suppose that there exists a real number $M>0$ such that

$$
g\left(t, x_{1}\right)-g\left(t, x_{2}\right) \leq \frac{M}{1+t^{q}}\left[\left(x_{1}-f\left(t, x_{1}\right)\right)-\left(x_{2}-f\left(t, x_{2}\right)\right)\right], \quad t \in J,
$$

for all $x_{1}, x_{2} \in \mathbb{R}$ with $x_{1} \geq x_{2}$, where $M T^{q} \leq \frac{1}{\Gamma(1-q)}$. Furthermore, if there exists a function $u \in C(J, \mathbb{R})$ such that

$$
\left\{\begin{array}{l}
D^{q}[v(t)-f(t, v(t))] \geq g(t, v(t)), \quad \text { a.e. } t \in J \\
v\left(t_{0}\right)>x_{0}
\end{array}\right.
$$

Then

$$
\rho(t) \leq v(t)
$$

for all $t \in J$, where $\rho$ is a minimal solution of FHDE (2.1) on $J$.

Note that Theorem 6.1 is useful to prove the boundedness and uniqueness of the solutions for FHDE (2.1) on $J$. A result in this direction is as follows.

Theorem 6.3 Assume that hypotheses $\left(\mathrm{A}_{0}\right)-\left(\mathrm{A}_{2}\right)$ hold. Suppose that there exists a function $G: J \times \mathbb{R}_{+} \rightarrow \mathbb{R}_{+}$such that

$$
\left|g\left(t, x_{1}\right)-g\left(t, x_{2}\right)\right| \leq G\left(t,\left|\left(x_{1}(s)-f\left(t, x_{1}(s)\right)\right)-\left(x_{2}(s)-f\left(t, x_{2}(s)\right)\right)\right|\right), \quad \text { a.e. } t \in J,
$$


for all $x_{1}, x_{2} \in \mathbb{R}$. If an identically zero function is the only solution of the differential equation

$$
D^{q} m(t)=G(t, m(t)) \text { a.e. } t \in J, m\left(t_{0}\right)=x_{0},
$$

then FHDE (2.1) has a unique solution on J.

Proof By Theorem 3.1, FHDE (2.1) has a solution defined on J. Suppose that there are two solutions $u_{1}$ and $u_{2}$ of FHDE (2.1) existing on $J$. Define a function $m: J \rightarrow \mathbb{R}$ by

$$
m(t)=\left|\left(u_{1}(t)-f\left(t, u_{1}(t)\right)\right)-\left(u_{2}(t)-f\left(t, u_{2}(t)\right)\right)\right| .
$$

As $D^{\alpha}(|x(t)|) \leq\left|D^{\alpha} x(t)\right|$ for $t \in J$, we have

$$
\begin{aligned}
D^{q} m(t) & =\left|D^{q}\left(u_{1}(t)-f\left(t, u_{1}(t)\right)\right)-D^{q}\left(u_{2}(t)-f\left(t, u_{2}(t)\right)\right)\right| \\
& =\left|g\left(t, u_{1}(t)\right)-g\left(t, u_{2}(t)\right)\right| \\
& \leq G\left(t,\left(u_{1}-f\left(t, u_{1}\right)\right)-\left(u_{2}-f\left(t, u_{2}\right)\right)\right) \\
& =G(t, m(t))
\end{aligned}
$$

for almost everywhere $t \in J$, and $m\left(t_{0}\right)=0$.

Now, we apply Theorem 6.1 with $f(t, x) \equiv 0$ to get that $m(t) \equiv 0$ for all $t \in J$. This gives

$$
u_{1}-f\left(t, u_{1}\right)=\left(u_{2}-f\left(t, u_{2}\right)\right.
$$

for all $t \in J$. Then we can get $u_{1}=u_{2}$ in view of hypothesis $\left(\mathrm{A}_{0}\right)$. This completes the proof.

\section{Existence of extremal solutions in a vector segment}

Sometimes it is desirable to have knowledge of the existence of extremal positive solutions for FHDE (2.1) on $J$. In this section, we prove the existence of maximal and minimal positive solutions for FHDE (2.1) between the given upper and lower solutions on $J=\left[t_{0}, t_{0}+a\right]$. We use a hybrid fixed point theorem of Dhage [26] in ordered Banach spaces for establishing our results. We need the following preliminaries in what follows.

A nonempty closed set $K$ in a Banach algebra $X$ is called a cone with vertex 0 if

(i) $K+K \subseteq K$,

(ii) $\lambda K \subseteq K$ for $\lambda \in \mathbb{R}, \lambda \geq 0$,

(iii) $(-K) \cap K=0$, where 0 is the zero element of $X$,

(iv) A cone $K$ is called positive if $K \circ K \subseteq K$, where $\circ$ is a multiplication composition in $X$.

We introduce an order relation ' $\leq$ ' in $X$ as follows. Let $x, y \in X$. Then $x \leq y$ if and only if $y-x \in K$. A cone $K$ is called normal if the norm $\|\cdot\|$ is semi-monotone increasing on $K$, that is, there is a constant $N>0$ such that $\|x\| \leq N\|y\|$ for all $x, y \in K$ with $x \leq y$. It is known that if the cone $K$ is normal in $X$, then every order-bounded set in $X$ is norm-bounded. The details of cones and their properties appear in Heikkilä and Lakshmikantham [31]. 
Lemma 7.1 [26] Let $K$ be a positive cone in a real Banach algebra $X$ and let $u_{1}, u_{2}, v_{1}, v_{2} \in$ $K$ be such that $u_{1} \leq v_{1}$ and $u_{2} \leq v_{2}$. Then $u_{1} u_{2} \leq v_{1} v_{2}$.

For any $a, b \in X$, the order interval $[a, b]$ is a set in $X$ given by

$$
[a, b]=\{x \in X: a \leq x \leq b\}
$$

Definition 7.1 A mapping $T:[a, b] \rightarrow X$ is said to be nondecreasing or monotone increasing if $x \leq y$ implies $T x \leq T y$ for all $x, y \in[a, b]$.

We use the following fixed point theorems of Dhage [27] for proving the existence of extremal solutions for IVP (2.1) under certain monotonicity conditions.

Lemma 7.2 [27] Let $K$ be a cone in a Banach algebra $X$ and let $a, b \in X$ be such that $a \leq b$. Suppose that $A, B:[a, b] \rightarrow K$ are two nondecreasing operators such that

(a) $A$ is a nonlinear contraction,

(b) B is completely continuous,

(c) $A x+B x \in[a, b]$ for each $x \in[a, b]$.

Further, if the cone $K$ is positive and normal, then the operator equation $A x+B x=x$ has a least and a greatest positive solution in $[a, b]$.

We equip the space $C(J, \mathbb{R})$ with the order relation $\leq$ with the help of a cone $K$ defined by

$$
K=\{x \in C(J, \mathbb{R}): x(t) \geq 0, \forall t \in J\}
$$

It is well known that the cone $K$ is positive and normal in $C(J, \mathbb{R})$. We need the following definitions in what follows.

Definition 7.2 A function $a \in C(J, \mathbb{R})$ is called a lower solution of FHDE (2.1) defined on $J$ if it satisfies (4.3). Similarly, a function $a \in C(J, \mathbb{R})$ is called an upper solution of FHDE (2.1) defined on $J$ if it satisfies (4.4). A solution to FHDE (2.1) is a lower as well as an upper solution for FHDE (2.1) defined on $J$ and vice versa.

We consider the following set of assumptions:

$\left(\mathrm{B}_{0}\right) f: J \times \mathbb{R} \rightarrow \mathbb{R}^{+}-\{0\}, g: J \times \mathbb{R} \rightarrow \mathbb{R}^{+}$.

$\left(\mathrm{B}_{1}\right)$ FHDE (2.1) has a lower solution $a$ and an upper solution $b$ defined on $J$ with $a \leq b$.

$\left(\mathrm{B}_{2}\right)$ The function $x \rightarrow x-f(t, x)$ is increasing in the interval $\left[\min _{t \in J} a(t), \max _{t \in J} b(t)\right]$ almost everywhere for $t \in J$.

$\left(\mathrm{B}_{3}\right)$ The functions $f(t, x)$ and $g(t, x)$ are nondecreasing in $x$ almost everywhere for $t \in J$.

$\left(\mathrm{B}_{4}\right)$ There exists a function $k \in L^{1}(J, \mathbb{R})$ such that $g(t, b(t)) \leq k(t)$.

We remark that hypothesis $\left(\mathrm{B}_{4}\right)$ holds in particular if $f$ is continuous and $g$ is $L^{1}$ Carathéodory on $J \times \mathbb{R}$.

Theorem 7.1 Suppose that assumptions $\left(\mathrm{A}_{1}\right)$ and $\left(\mathrm{B}_{0}\right)-\left(\mathrm{B}_{4}\right)$ hold. Then FHDE $(2.1)$ has a minimal and a maximal positive solution defined on $J$. 
Proof Now, FHDE (2.1) is equivalent to integral equation (3.5) defined on $J$. Let $X=$ $C(J, \mathbb{R})$. Define two operators $A$ and $B$ on $X$ by (3.6) and (3.7) respectively. Then the integral equation (3.5) is transformed into an operator equation $A x(t)+B x(t)=x(t)$ in the Banach algebra $X$. Notice that hypothesis $\left(\mathrm{B}_{0}\right)$ implies $A, B:[a, b] \rightarrow K$. Since the cone $K$ in $X$ is normal, $[a, b]$ is a norm bounded set in $X$. Now it is shown, as in the proof of Theorem 3.1, that A is a Lipschitzian with the Lipschitz constant $L$ and $B$ is a completely continuous operator on $[a, b]$. Again, hypothesis $\left(\mathrm{B}_{3}\right)$ implies that $A$ and $B$ are nondecreasing on $[a, b]$. To see this, let $x, y \in[a, b]$ be such that $x \leq y$. Then, by hypothesis $\left(\mathrm{B}_{3}\right)$,

$$
A x(t)=f(t, x(t)) \leq f(t, y(t))=A y(t)
$$

for all $t \in J$. Similarly, we have

$$
\begin{aligned}
B x(t) & =x_{0}-f\left(t_{0}, x_{0}\right)+\frac{1}{\Gamma(q)} \int_{t_{0}}^{t}(t-s)^{q-1} g(s, x(s)) d s \\
& \leq x_{0}-f\left(t_{0}, x_{0}\right)+\frac{1}{\Gamma(q)} \int_{t_{0}}^{t}(t-s)^{q-1} g(s, y(s)) d s \\
& =B y(t)
\end{aligned}
$$

for all $t \in J$. So, $A$ and $B$ are nondecreasing operators on $[a, b]$. Lemma 7.1 and hypothesis $\left(\mathrm{B}_{3}\right)$ together imply that

$$
\begin{aligned}
a(t) & \leq x_{0}-f\left(t_{0}, x_{0}\right)+f(a, a(t)) \frac{f(t, a(t))}{\Gamma(q)} \int_{t_{0}}^{t}(t-s)^{q-1} g(s, x(s)) d s \\
& \leq x_{0}-f\left(t_{0}, x_{0}\right)+f(t, x(t))+\frac{f(t, x(t))}{\Gamma(q)} \int_{t_{0}}^{t}(t-s)^{q-1} g(s, x(s)) d s \\
& \leq x_{0}-f\left(t_{0}, x_{0}\right)+f(t, b(t))+\frac{f(t, b(t))}{\Gamma(q)} \int_{t_{0}}^{t}(t-s)^{q-1} g(s, x(s)) d s \\
& \leq b(t)
\end{aligned}
$$

for all $t \in J$ and $x \in[a, b]$. As a result, $a(t) \leq A x(t)+B x(t) \leq b(t)$ for all $t \in J$ and $x \in[a, b]$. Hence, $A x+B x \in[a, b]$ for all $x \in[a, b]$.

Now, we apply Lemma 7.2 to the operator equation $A x B x=x$ to yield that FHDE (2.1) has a minimal and a maximal positive solution in $[a, b]$ defined on $J$. This completes the proof. 
Science Foundation (ZR2012AM009, ZR2010AL002, ZR2011 AL007), also supported by Natural Science Foundation of Educational Department of Shandong Province (J11LA01).

\section{Received: 30 November 2012 Accepted: 22 January 2013 Published: 11 February 2013}

\section{References}

1. Miller, KS, Ross, B: An Introduction to the Fractional Calculus and Fractional Differential Equation. Wiley, New York (1993)

2. Oldham, KB, Spanier, J: The Fractional Calculus. Academic Press, New York (1974)

3. Podlubny, I: Fractional Differential Equations, Mathematics in Science and Engineering. Academic Press, New York (1999)

4. Samko, SG, Kilbas, AA, Marichev, Ol: Fractional Integral and Derivative, Theory and Applications. Gordon \& Breach, New York (1993)

5. Zhao, Y, Sun, S, Han, Z, Li, Q: The existence of multiple positive solutions for boundary value problems of nonlinear fractional differential equations. Commun. Nonlinear Sci. Numer. Simul. 16, 2086-2097 (2011)

6. Zhao, Y, Sun, S, Han, Z, Li, Q: Positive solutions to boundary value problems of nonlinear fractional differential equations. Abstr. Appl. Anal. 2011, 1-16 (2011)

7. Zhou, Y, Jiao, F, Li, J: Existence and uniqueness for $p$-type fractional neutral differential equations. Nonlinear Anal. TMA 71, 2724-2733 (2009)

8. Zhou, Y, Jiao, F, Li, J: Existence and uniqueness for fractional neutral differential equations with infinite delay. Nonlinear Anal. TMA 71, 3249-3256 (2009)

9. Zhou, Y, Jiao, F: Nonlocal Cauchy problem for fractional evolution equations. Nonlinear Anal., Real World Appl. 11, 4465-4475 (2010)

10. Wang, J, Zhou, Y: A class of fractional evolution equations and optimal controls. Nonlinear Anal., Real World Appl. 12, 262-272 (2011)

11. Agarwal, RP, Zhou, Y, He, Y: Existence of fractional neutral functional differential equations. Comput. Math. Appl. 59 1095-1100 (2010)

12. $\mathrm{Li}, \mathrm{CF}, \mathrm{LuO}, \mathrm{XN}$, Zhou, Y: Existence of positive solutions of boundary value problem for fractional differential equations. Comput. Math. Appl. 59, 1363-1375 (2010)

13. Diethelm, K: The Analysis of Fractional Differential Equations. Springer, Berlin (2010)

14. Sun, S, Zhao, Y, Han, Z, Xu, M: Uniqueness of positive solutions for boundary value problems of singular fractional differential equations. Inverse Probl. Sci. Eng. 20, 299-309 (2012)

15. Lakshmikantham, V, Vatsala, AS: Basic theory of fractional differential equations. Nonlinear Anal. TMA 69, 2677-2682 (2008)

16. Lakshmikantham, V, Vatsala, AS: Theory of fractional differential inequalities and applications. Commun. Appl. Anal. 11, 395-402 (2007)

17. Lakshmikantham, V: Theory of fractional functional differential equations. Nonlinear Anal. TMA 69, 3337-3343 (2008)

18. Lakshmikantham, V, Devi, JV: Theory of fractional differential equations in Banach space. Eur. J. Pure Appl. Math. 1, 38-45 (2008)

19. Kilbas, AA, Srivastava, HH, Trujillo, JJ: Theory and Applications of Fractional Differential Equations. Elsevier, Amsterdam (2006)

20. Caputo, M: Linear models of dissipation whose Q is almost independent II. Geophys. J. R. Astron. Soc. 13, 529-539 (1967)

21. Diethelm, K, Ford, NJ: Analysis of fractional differential equations. J. Math. Anal. Appl. 265, 229-248 (2002)

22. Diethelm, K, Ford, NJ: Multi-order fractional differential equations and their numerical solution. Appl. Math. Comput. $154,621-640$ (2004)

23. Dhage, BC: On $\alpha$-condensing mappings in Banach algebras. Math. Stud. 63, 146-152 (1994)

24. Dhage, BC, Lakshmikantham, V: Basic results on hybrid differential equations. Nonlinear Anal., Real World Appl. 4 , 414-424 (2010)

25. Dhage, BC, Jadhav, NS: Basic results in the theory of hybrid differential equations with linear perturbations of second type (submitted)

26. Dhage, BC: A nonlinear alternative in Banach algebras with applications to functional differential equations. Nonlinear Funct. Anal. Appl. 8, 563-575 (2004)

27. Dhage, BC: Fixed point theorems in ordered Banach algebras and applications. Panam. Math. J. 9, 93-102 (1999)

28. Lakshmikantham, V, Leela, S: Differential and Integral Inequalities. Academic Press, New York (1969)

29. Zhao, Y, Sun, S, Han, Z, Li, Q: Theory of fractional hybrid differential equations. Comput. Math. Appl. 62, 1312-1324 (2011)

30. Dhage, BC: A fixed point theorem in Banach algebras with applications to functional integral equations. Kyungpook Math. J. 44, 145-155 (2004)

31. Heikkilä, S, Lakshmikantham, V: Monotone Iterative Technique for Nonlinear Discontinuous Differential Equations. Dekker, New York (1994)

doi:10.1186/1687-2770-2013-23

Cite this article as: Lu et al.: Theory of fractional hybrid differential equations with linear perturbations of second type. Boundary Value Problems 2013 2013:23. 